\title{
MERITS OF SPACE VLBI MISSIONS FOR GEODYNAMICS
}

\author{
JOZSEF ADAM * and IVAN I. MUELLER \\ Department of Geodetic Science and Surveying, Ohio State University, \\ Columbus, Ohio, U.S.A. 43210-1247
}

\begin{abstract}
Two dedicated space VLBI projects are currently in preparation to launch one or more VLBI radio telescopes in orbit between 1993-1996. One in the Soviet Union called RADIOASTRON is already an approved and funded mission. The second one is a Japanese orbiting VLBI mission called VSOP. There is a. Western European mission with NASA participation in Phase A Study at ESA called QUASAT. Although this project was not approved by ESA at the end of 1988, it might still be taken into consideration during the next decade. In the meantime in 1986 a successful demonstration of space VLBI was made at the first attempt using the NASA TDRSS satellite.
\end{abstract}

It is expected that space VLBI will be a reality in the next decade. Orbiting radio telescopes will be used to make interferometric observations of extragalactic radio sources in conjunction with the major ground-based VLBI arrays in Europe, USA, Australia, Japan and the USSR. It is planned to determine the orbits of these radio telescopes in space with high accuracy by the missions themselves and possibly using additional tracking systems (e.g., GPS, PRARE). The main goals of all space VLBI projects are to carry out astrophysical investigations. However, astrophysical space VLBI data may be used for geodesy and geodynamics as well. Therefore we started to investigate potential possibilities of space VLBI missions for these areas. The space VLBI observables may be useful to improve Earth's gravity field and in the unification and connection of reference frames inherent in the space VLBI technique. Our work is to explore the feasibility of these potential applications and to provide sufficient background for the inclusion of space VLBI observables in geodetic data processing programs (e.g., GEODYN). In this presentation, the mathematical models for space VLBI observables suitable for covariance analysis are described. A summary of estimable parameters is included. Singularity problems arising from coordinate system definition, observability conditions and critical configurations will be studied. Finally, the scope for space VLBI in geodesy and geodynamics is outlined. Acknowledgement. This research is supported by NASA under Research Grant No.

NSG 5265.

* J. Adams is on leave from Satellite Geodetic Observatory, Hungary.

Y. Kondo (ed.), Observatories in Earth Orbit and Beyond, 507.

(C)1990 Kluwer Academic Publishers. Printed in The Netherlands. 